\title{
Erratum to: Joint application-architeture design space exploration of multimedia applications on many-core platforms - an experimental analysis
}

\author{
Maryam Moghadas ${ }^{1}$ - Hossein Afshari ${ }^{2}$. \\ Mahmoud Reza Hashemi ${ }^{1}$
}

Published online: 2 September 2015

(C) Springer Science+Business Media New York 2015

\section{Erratum to: Multimed Tools Appl \\ DOI 10.1007/s11042-015-2854-4}

Due to an error during typesetting, the data "Member IEEE" was incorrectly added in the author group in the original version of this article. The correct authors' list is given above. The original version was corrected.

The online version of the original article can be found at http://dx.doi.org/10.1007/s11042-015-2854-4.

\author{
Maryam Moghadas \\ mmoghadas@ut.ac.ir \\ Hossein Afshari \\ hossein.afshari@a3.epfl.ch \\ Mahmoud Reza Hashemi \\ rhashemi@ut.ac.ir
}

1 Multimedia Processing Laboratory, School of Electrical and Computer Engineering College of Engineering, University of Tehran, Tehran, Iran

2 EPFL Alumni, EPFL University, Lausanne, Switzerland 